\title{
Prediction of axillary response after neoadjuvant chemotherapy in clinical node positive breast cancer
}

\author{
Weizhen Zheng ${ }^{1 \#}$, Pengpeng Zhou ${ }^{2,3 \#}$, Yanbing Liu ${ }^{3}$, Ying Liang ${ }^{3,4}$, Yongsheng Wang ${ }^{3}$ \\ ${ }^{1}$ Shandong Provincial Hospital, Jinan, China; ${ }^{2}$ Shandong First Medical University \& Shandong Academy of Medical Sciences, Jinan, China; \\ ${ }^{3}$ Shandong Cancer Hospital and Institute, Shandong First Medical University and Shandong Academy of Medical Sciences, Jinan, China; ${ }^{4}$ Cheeloo \\ College of Medicine, Shandong University, Jinan, China \\ Contributions: (I) Conception and design: W Zheng, P Zhou; (II) Administrative support: Y Wang; (III) Provision of study materials or patients: Y \\ Liang; (IV) Collection and assembly of data: Y Liu; (V) Data analysis and interpretation: W Zheng, P Zhou; (VI) Manuscript writing: All authors; (VII) \\ Final approval of manuscript: All authors. \\ \#These authors contributed equally to this work. \\ Correspondence to: Yongsheng Wang. Shandong Cancer Hospital and Institute, Ji Yan Road 440, Jinan, Shandong Province, China. \\ Email: wangysh2008@aliyun.com.
}

Background: For clinical lymph node positive $(\mathrm{cN}+)$ breast cancer, the false negative rate of sentinel lymph node biopsy (SLNB) after neoadjuvant chemotherapy (NAC) is high. Prediction of axillary response after NAC may provide a better way of patient selection. Our study was designed to evaluate factors associated with axillary pathologic complete response (ypN0) after NAC, and to assess the accuracy of the published Olga Kantor predictive model.

Methods: A total of 406 patients with $\mathrm{cN}+$ breast cancer were enrolled in this study. All patients had received full courses of NAC before undergoing axillary lymph node dissection (ALND). Univariate analyses and multivariate analysis were performed to explore independent predictors of ypN0. Then the Olga Kantor model were validated by the data of patients enrolled. The Olga Kantor model is not ideal because the pathological breast tumor response was not available before surgery, the clinical breast tumor response was assessed in our study as a modification. The accuracy of the validation and modification of Olga Kantor model were assessed by the area under receiver operating characteristic (ROC) curve (AUC).

Results: Age $(\mathrm{P}=0.004)$, molecular subtype $(\mathrm{P}=0.000)$, tumor grade $(\mathrm{P}=0.006)$, clinical tumor response $(\mathrm{P}=0.000)$ and $\mathrm{Ki}-67(\mathrm{P}=0.009)$ were correlated with ypN0. Age, molecular subtype and the clinical tumor response were independent predictors of ypN0 $(\mathrm{P}<0.05)$. In validation and modification model, the AUC values were 0.795 and 0.789 , respectively, there were no significant differences between the two models $(\mathrm{P}=0.536)$. For model score $\leq 3,4-7$ and $\geq 8$ in the modification model, the ypN0 rate were $3.9 \%(2 / 51)$, $22.5 \%(59 / 262)$ and $67.7 \%(63 / 93)$, respectively.

Conclusions: The Olga Kantor predictive model had high accuracy predicting ypN0 after NAC. Our modification model achieved the same predictive efficiency but is more feasible for clinical practice. Patients with higher scores were more likely to achieve ypN0, so SLNB might be a better way than ALND. However, more patient data and multicenter cohort trials are needed to verify the study.

Keywords: Axillary pathologic complete response (ALND); breast cancer; predictive model; sentinel lymph node biopsy (SLNB)

Submitted Dec 17, 2020. Accepted for publication Apr 23, 2021.

doi: $10.21037 /$ tcr-20-3454

View this article at: https://dx.doi.org/10.21037/tcr-20-3454 


\section{Introduction}

Sentinel lymph node biopsy (SLNB) has replaced axillary lymph node dissection (ALND) as the standard surgery for patients with clinically node-negative (cN0) breast cancer. This procedure can significantly reduce the incidence of upper limb lymphedema, paresthesia, and dyskinesia $(1,2)$. In recent years, SLNB has also been suggested for clinically node-positive $(\mathrm{cN}+)$ patients who become clinically node-negative (ycN0) after neoadjuvant chemotherapy (NAC) (3). The Sentina and ACOSOG 1071 trials showed that the false-negative rate (FNR) could be reduced to less than $10 \%$ if SLNB was performed under certain circumstances, including using double tracers and detecting at least 3 sentinel lymph nodes (SLNs) $(4,5)$. Based on these trials, SLNB after NAC has been incorporated into the National Comprehensive Cancer Network (NCCN) guidelines and St. Gallen International Expert Consensus $(6,7)$. However, the overall FNR is still high in clinical practice, and ALND remains a standard option for these patients. False-negative patients with residual disease may receive inadequate treatment. Therefore, optimal patient selection is of great importance not only to avoid unnecessary ALND but also to guide adjuvant treatment after surgery. More appropriate candidates for SLNB should be patients who achieve axillary pathologic complete response (ypN0) upon completing NAC. Given these concerns, Olga Kantor et al. developed a model to predict which patients have the maximum likelihood of achieving ypN0. The areas under the receiver operating characteristic (ROC) curves of the testing and validation cohorts in this study were 0.781 and 0.788 , respectively (8). The purpose of our study was to explore the independent predictors of ypN0 and to verify the accuracy of the Olga Kantor predictive model. We present the following article in accordance with the TRIPOD reporting checklist (available at https://dx.doi.org/10.21037/tcr-20-3454).

\section{Methods}

\section{Patient data}

The patient data were generated from clinical and pathologic data available at Shandong Cancer Hospital from April 2015 to May 2019, and 406 patients were included in this retrospective study. All recruited patients had been diagnosed with pathological invasive breast cancer through core-needle biopsy (core needle size: 12-14 G, 3-5 tissue strips), and their axillary lymph nodes had been proven positive by either fine-needle aspiration or coreneedle biopsy. In addition, all patients had finished full courses of NAC with anthracyclines and/or taxane before routine breast surgery (including breast-conserving surgery, mastectomy, and breast reconstruction) and standard ALND. The study was conducted in accordance with the Declaration of Helsinki (as revised in 2013). The study was approved by the Ethics Committee of Shandong Cancer Hospital (No. SDTHEC 20110324), and individual consent for this retrospective analysis was waived. Patient data, such as patient demographics, tumor size, stage, molecular subtype, and clinical and pathological breast tumor response, were recorded. Hormone receptor (HR) positivity was defined as either estrogen or progesterone receptor $\geq 1 \%$ in tumor tissue. Her-2 positivity was defined as immunohistochemistry or fluorescence in situ hybridization (FISH) detection positivity. The standard ALND required that a minimum of 10 lymph nodes be dissected and examined on pathology. The clinical breast tumor response was evaluated by MRI tumor size before and after NAC, while pathological tumor response was assessed by comparing the preoperative imaging size with the postoperative pathological size. The breast tumor response could be divided into 3 subgroups according to RECIST version 1.1 (9): both progressive disease (PD) and stable disease (SD) in RECIST were considered no response, partial response (PR) was considered partial tumor response, and complete response (CR) was considered complete tumor response. Pathologic complete response (pCR) refers to no residual tumor cells (including isolated tumor cells) in the primary breast and axillary lymph node specimens by pathological examination.

\section{Validation of the ypNO predictive model}

Univariate analysis was used to determine the factors associated with ypN0 after NAC, and then the significant factors were included in a multivariate analysis to explore independent predictors of ypN0. The data of all 406 included patients were then input into the Olga Kantor predictive model for validation, the model score was calculated for each patient according to Table 1, and the predictive efficiency was assessed. However, considering that the Olga Kantor model is not ideal because the pathological breast tumor response was not available at the time of surgical decision-making, the actual clinical breast tumor response was assessed in our study by a surrogate by comparing the MRI breast tumor size before and after 
Table 1 Model score calculation in Olga Kantor model

\begin{tabular}{|c|c|}
\hline Variable & Assigned point score \\
\hline \multicolumn{2}{|l|}{ Age (year) } \\
\hline$\geq 50$ & 1 \\
\hline$<50$ & 1.5 \\
\hline \multicolumn{2}{|l|}{ Molecular subtype } \\
\hline HR+/HER2- & 1 \\
\hline HR-/HER2- & 3 \\
\hline $\mathrm{HR}+/ \mathrm{HER} 2+$ & 4 \\
\hline HR-/HER2+ & 5 \\
\hline \multicolumn{2}{|l|}{ Tumor grade } \\
\hline Grade1/2 & 1 \\
\hline Grade 3 & 1.5 \\
\hline \multicolumn{2}{|l|}{ Tumor histology } \\
\hline Lobular/mixed & 1 \\
\hline Ductal & 1.5 \\
\hline \multicolumn{2}{|l|}{ cN stage } \\
\hline $\mathrm{cN} 2 / \mathrm{N} 3$ & 1 \\
\hline $\mathrm{cN} 1$ & 1.5 \\
\hline \multicolumn{2}{|l|}{ Breast tumor response } \\
\hline No response & 0 \\
\hline Partial tumor response & 2 \\
\hline Complete tumor response & 4 \\
\hline Total $^{\star}$ & $5-15$ \\
\hline
\end{tabular}

*, The model was adjusted to a 1-10 numeric scale by subtracting 5 from the total score. Reprinted by permission from Springer Nature Customer Service Centre GmbH, Springer Nature, Annals of Surgical Oncology (8).

NAC as a modification. The model score and the predictive efficiency of the modification model were also analyzed (8).

\section{Statistical analysis}

Statistical analysis was performed by using SPSS 22.0 (IBM Company, Armonk, NY). Chi square (v2) tests were performed to examine univariate factors associated with ypN0, and the significant factors were then included in multivariate logistic regression to determine the independent predictive factors for ypN0 after NAC. The model score was calculated for each patient, and model accuracy was tested by the area under the curve (AUC). The $Z$ test was performed to analyze the difference in AUC between the validation and modification models. The ypN0 rate was analyzed according to model score. $\mathrm{P}<0.05$ was considered statistically significant.

\section{Results}

\section{Clinical data}

From April 2015 to May 2019, 406 patients with cN+ breast cancer were enrolled in this study. All patients had received full courses of NAC before undergoing ALND, and there were no missing data for the enrolled patients. The median age was 56 years (28-70 years). Of these patients, 124 (30.5\%) had a complete pathologic nodal response after full courses of NAC.

\section{Analysis of ypNO predictors}

Data from patients who achieved ypN0 were compared to those with residual positive nodes (ypN+). In 406 patients, age $(\mathrm{P}=0.004)$, molecular subtype $(\mathrm{P}=0.000)$, tumor grade $(\mathrm{P}=0.006)$, clinical tumor response $(\mathrm{P}=0.000)$ and $\mathrm{Ki}-67$ $(\mathrm{P}=0.009)$ were correlated with ypN0 after NAC (Table 2). Then, the related factors were entered into a multivariate regression, which indicated that age $(\mathrm{OR}=7.457,95 \% \mathrm{CI}$ : 2.545-21.852), molecular subtype $(\mathrm{OR}=1.373,95 \% \mathrm{CI}$ : $1.167-1.616)$ and clinical tumor response $(\mathrm{OR}=3.254,95 \%$ CI: 2.462-4.301) were independent predictors of ypN0 $(\mathrm{P}<0.05)$ (Table 3).

\section{Validation and modification of the Olga Kantor predictive model}

The data of all 406 patients were input into the Olga Kantor predictive model for validation. Then, a modification was made by using the clinical tumor response as a substitute for the pathological tumor response. The model scores for enrolled patients in the validation and modification models were determined (Table 4). In both models, an increasing score was correlated with a stepwise increase in the ypNO rate. The overall trend of ypN0 percentage in our study is shown in Figure 1. The AUC values of the validation and modification models were 0.795 and 0.789 , respectively (Figure 2) and were not significantly different ( $\mathrm{P}=0.536)$. In the modification model, the ypN0 rate for patients with a model score no greater than 3 was $3.9 \%(2 / 51)$, the rate for 
Table 2 Univariate analysis of ypN0

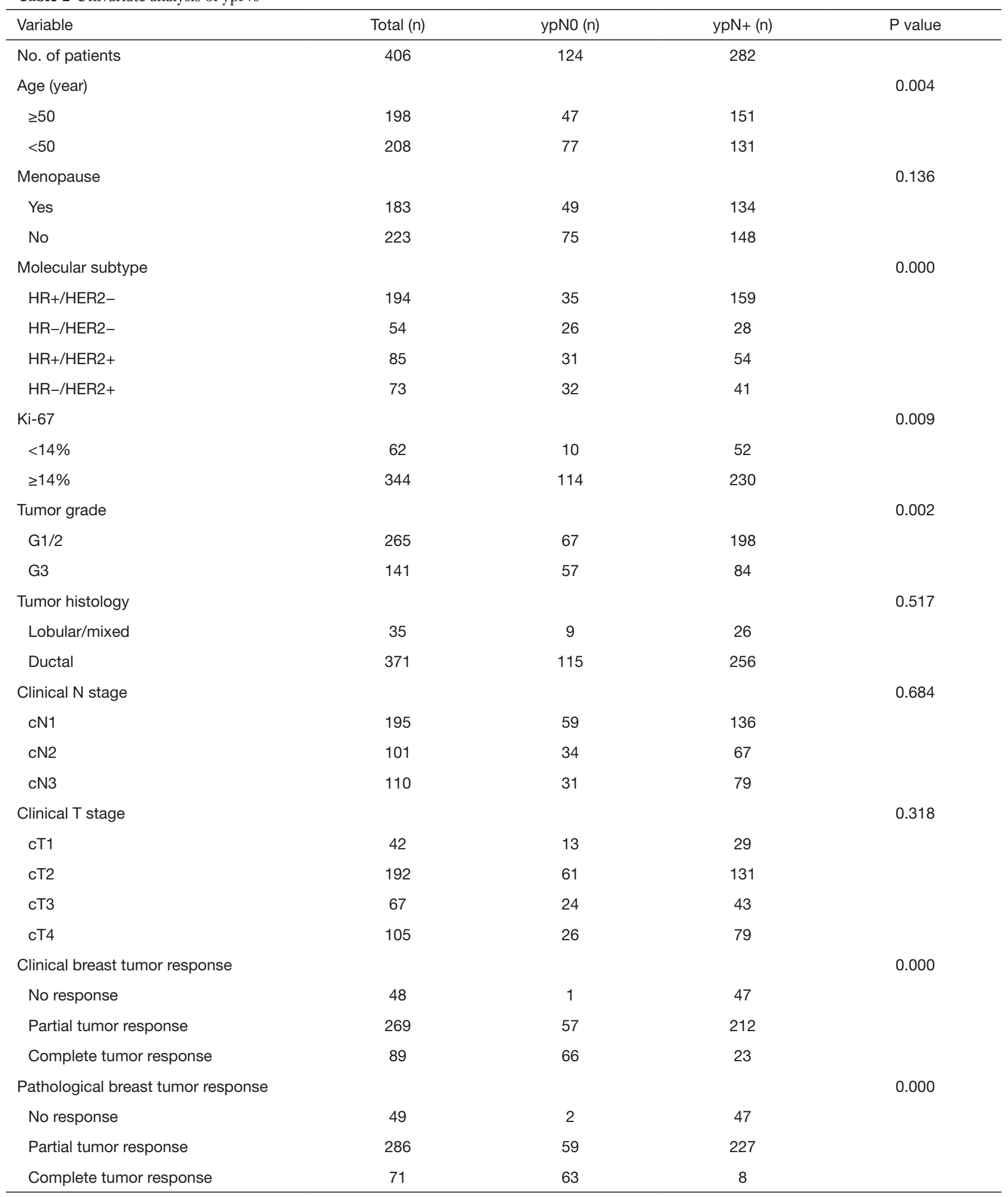


Table 3 Multivariate logistic regression analysis of ypN0

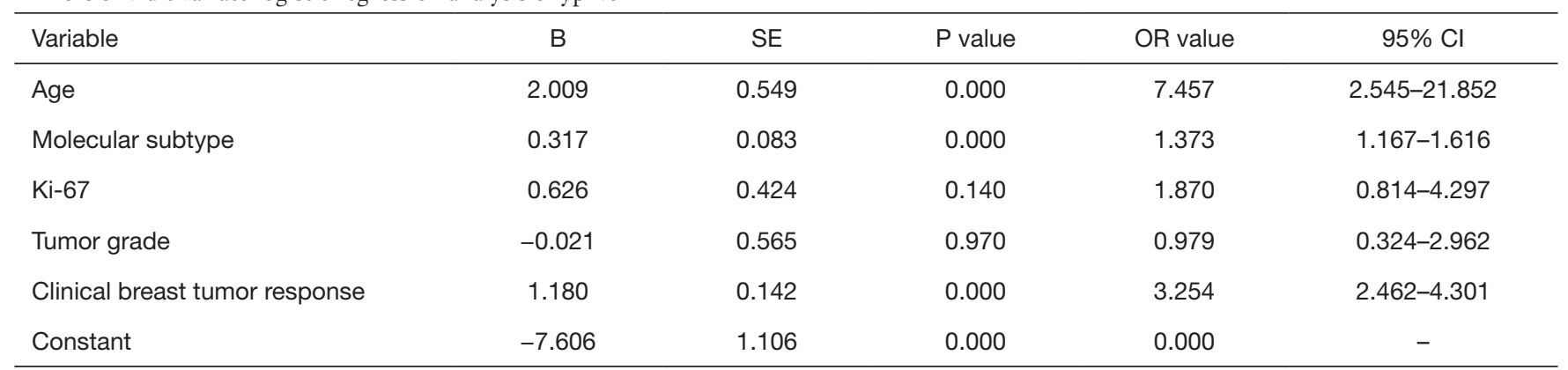

B, partial regression coefficient; SE, standard error; OR, odds ratio; $95 \% \mathrm{CI}, 95 \%$ confidence interval.

Table 4 Model score for patients in validation/modification model

\begin{tabular}{|c|c|c|c|c|c|}
\hline Total score & Model score & \multicolumn{2}{|c|}{ ypNo (n) } & \multicolumn{2}{|c|}{ ypNo (\%) } \\
\hline $5-5.5$ & 1 & $0 / 2$ & $0 / 5$ & $0.0 \%$ & $0.0 \%$ \\
\hline $6-6.5$ & 2 & $1 / 25$ & $0 / 18$ & $4.0 \%$ & $0.0 \%$ \\
\hline $7-7.5$ & 3 & $1 / 31$ & $2 / 28$ & $3.2 \%$ & $7.1 \%$ \\
\hline $9-9.5$ & 5 & $4 / 15$ & $3 / 17$ & $26.7 \%$ & $17.6 \%$ \\
\hline $10-10.5$ & 6 & $21 / 57$ & $21 / 64$ & $36.8 \%$ & $32.8 \%$ \\
\hline $11-11.5$ & 7 & $23 / 68$ & $18 / 60$ & $33.8 \%$ & $30.0 \%$ \\
\hline $12-12.5$ & 8 & $27 / 48$ & $27 / 47$ & $56.3 \%$ & $57.4 \%$ \\
\hline
\end{tabular}

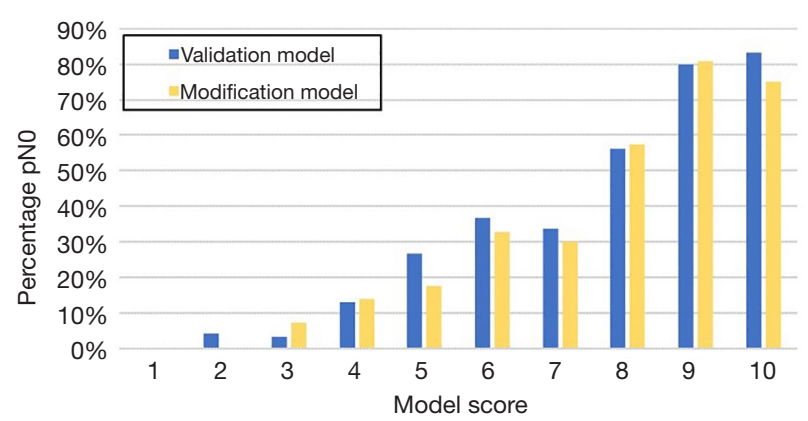

Figure 1 Percentage of ALND in validation and modification model. Adapted by permission from Springer Nature Customer Service Centre GmbH, Springer Nature, Annals of Surgical Oncology (8). patients with a model score of 4-7 was $22.5 \%$ (59/262), and the rate was $67.7 \%(63 / 93)$ when the model score was $\geq 8$.

\section{Discussion}

NAC is the standard treatment for patients with locally advanced breast cancer. A response of the tumor to chemotherapy increases the chance of breast-conserving surgery (10). Pathological complete response (pCR) after NAC is considered a sign of long-term survival benefit. The survival rate of patients who achieve pCR after NAC is significantly higher than that of patients with residual positive disease (11). Approximately $40-75 \%$ of patients with $\mathrm{cN}+$ disease achieve axillary pCR after NAC 


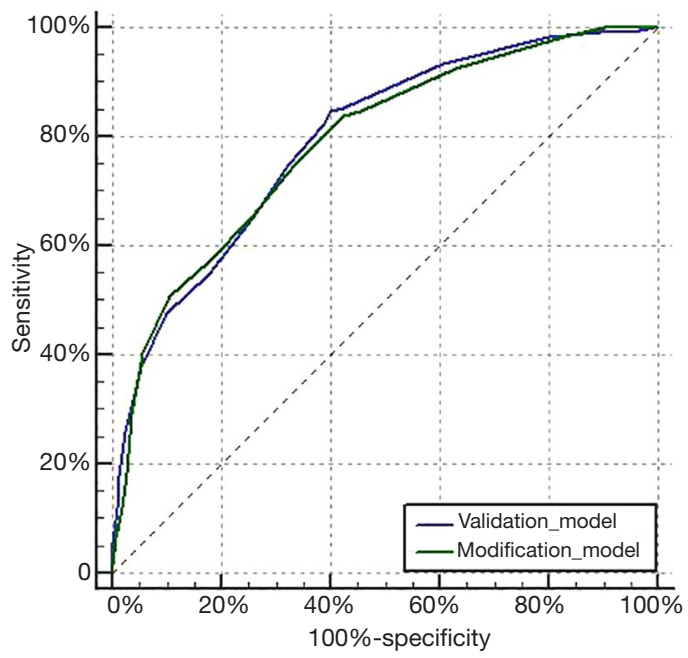

Figure 2 ROC curves for validation and modification model. The AUC values of the validation and modification models were 0.795 and $0.789(\mathrm{P}=0.536)$.

(12-14). In these patients, regular surgery for ALND would be an overtreatment that has no clear benefit but prolongs hospital stay and increases the incidence of morbidities (such as lymphedema). However, the axillary response to chemotherapy can only be evaluated through imaging examinations before surgery. Therefore, the ideal candidate for SLNB after NAC is a $\mathrm{cN}+$ patient who becomes clinically node-negative after NAC, the feasibility of which has been proven by the Sentina and ACOSOG 1071 trials $(4,5)$. However, the FNR of SLNB after NAC remains too high for widespread acceptance, which indicates that improvement is needed for the further application of SLNB in clinical practice. The SLNB technique could be improved by implanting markers in positive nodes before NAC, using double tracers and detecting $\geq 3$ SLNs in surgery. However, more important are changes in patient selection to increase sensitivity. More appropriate candidates are patients who are more likely to develop ypN0 upon completing NAC. Given these concerns, several studies have explored predictive models of ypN0 after NAC for better patient selection (8,15-17). Kantor et al. retrospectively analyzed data from 19,115 patients. Age, molecular subtype, tumor grade, tumor histology, $\mathrm{cN}$ stage, and pathologic tumor response were all significant independent predictors of ypN0 after NAC. The AUC values of the testing cohort $(\mathrm{n}=13,396)$ and validation cohort $(\mathrm{n}=5,719)$ in this predictive model were 0.781 and 0.788 , respectively (8). The study showed that patients with higher scores were more likely to achieve ypN0 and therefore were more suitable candidates for SLNB than ALND. Compared with other models, the Olga Kantor model has the advantage of a larger cohort and higher predictive accuracy. Therefore, this model was chosen for validation in our study.

In the Olga Kantor predictive model, all patient data were derived from the National Cancer Data Base NCDB database (18), which does not collect data on clinical tumor size after NAC; thus, the breast tumor response was assessed by comparing the clinical tumor size before NAC with the pathological tumor size after surgery. This model is conspicuously not applicable in clinical practice because pathological results are generally unavailable at the time of surgical decision-making. Therefore, we attempted to demonstrate that this model is also applicable when the real clinical tumor response is taken into account so that the axillary status can be predicted preoperatively. If the model is also accurate with the clinical tumor response, it may be possible to finally implement the model in clinical practice. Nevertheless, using the actual clinical response could produce a distinct predictive model that generates more accurate predictions. Since our sample size is too small, the data are far from sufficient to build a new model. Future studies might focus on developing new models more in line with clinical practice.

In our study, when we analyzed factors related to ypN0, we also used the clinical tumor response as the standard for evaluating the tumor response to NAC. We verified that age, molecular subtype, and breast tumor response were independent predictors of ypN0. However, tumor grade, tumor histology and clinical $\mathrm{N}$ stage, which were also independent predictors in the Olga Kantor model, did not show statistical significance in our study. This might be because the sample size of our study was much smaller $(n=350)$ than that of the Olga Kantor model $(n=19,115)$; there might also be an influence of regional differences. Moreover, the difference might be due to the method of assessing tumor response, as the influence coefficient of the pathological tumor response might be more significant than that of the clinical tumor response.

The validation of the Olga Kantor predictive model showed that the AUC was 0.795, indicating high accuracy of this model in our center. For the modification model, the AUC was 0.789. No significant difference in AUC was observed between the two models $(\mathrm{P}=0.536)$. Therefore, the modification model has the same predictive efficiency but is more in line with clinical practice.

Our study also attempted to address some other 
limitations of the Olga Kantor predictive model. First, we only selected patients who had completed full courses of anthracyclines and taxane-based neoadjuvant chemotherapy, whereas for the Olga Kantor predictive model, patients were recruited regardless of the specific chemotherapy regimens and number of cycles of therapy, which might lead to lower predictive effectiveness. Second, we analyzed several factors that were not included in the Olga Kantor predictive model, including Ki-67 and menstrual status, and found that none were independent predictors of ypN0 (Table 3).

The prediction of ypN0 after NAC can provide suggestions for selecting patients suitable for SLNB after NAC. In our modification model, the ypN0 rate in patients with a model score $\leq 3$ was $3.9 \%(2 / 51)$. For this group of patients, ALND might be a more appropriate selection. The ypN0 rate in patients with model scores of 4-7 was $22.5 \%$ (59/262). For these patients, SLNB might be feasible, particularly if markers are placed in the positive node before NAC, double tracers are used and more than two SLNs are detected to reduce the FNR. The ypN0 rate in patients with a model score $\geq 8$ was $67.7 \%$ (63/93). In these patients, the FNR of SLNB may be low enough to allow SLNB to be performed directly; thus, SLNB might be a better choice than ALND. It is worth noting that the ypN0 rate in patients who achieved a total score of 15 was $100 \%$ $(2 / 2)$. We expect that these patients can even avoid axillary surgery. The SOUND (19) and ASICS clinical trials have begun studying the possibility of avoiding axillary surgery in early breast cancer patients with negative preoperative axillary assessment (cN0). The results of these two trials may lay the foundation for avoiding axillary surgery in $\mathrm{cN}+$ patients. However, only 2 patients in our study achieved a total score of 15 , and this sample size is too small to provide valuable evidence for guiding axillary surgery options.

Our study also has several limitations. First, because the cohort of our study was small ( $<500$ patients), recommendations cannot be made based on the results of our study, and we can only give suggestions on which axillary surgery might be more appropriate according to the model score. There have been no reports of the longterm outcomes of $\mathrm{cN}+$ patients treated with NAC who did not undergo ALND. Therefore, whether oncologic safety is guaranteed when ALND is omitted is unknown, and further study is required to prove the clinical safety of omitting ALND. Second, the real clinical tumor response in this study was assessed by comparing the MRI tumor size before and after NAC. However, not all institutions routinely perform MRI before and after NAC. Ultrasound can be a replacement, but its accuracy is limited. As a method of avoiding ALND, targeted axillary dissection (TAD), which includes sentinel lymph node dissection and selective localization and removal of clipped nodes, has also been researched in recent years $(20,21)$. A recent review comparing the accuracy of SLNB, MRI and TAD found that TAD was the most accurate (22). The proposed predictive model for ypN0 after NAC can be used in TAD to further reduce the FNR.

\section{Conclusions}

The predictive model for $\mathrm{ypN} 0$ after $\mathrm{NAC}$ for $\mathrm{cN}+$ patients can provide reasonable patient selection for SLNB. We validated the high accuracy of the Olga Kantor model, and our modification model achieved the same predictive accuracy but is more suitable for clinical practice. Patients with higher scores were more likely to achieve ypN0 and therefore are more suitable candidates for SLNB than ALND. However, related research on this topic is insufficient, and more patient data and multicenter cohort trials are needed to verify these conclusions.

\section{Acknowledgments}

We thank AJE Company (https://www.aje.com/) for editing this manuscript.

Funding: None.

\section{Footnote}

Reporting Checklist: The authors have completed the TRIPOD reporting checklist. Available at https://dx.doi. org/10.21037/tcr-20-3454

Data Sharing Statement: Available at https://dx.doi. org/10.21037/tcr-20-3454

Peer Review File: Available at https://dx.doi.org/10.21037/ tcr-20-3454

Conflicts of Interest: All authors have completed the ICMJE uniform disclosure form (available at https://dx.doi. org/10.21037/tcr-20-3454). The authors have no conflicts of interest to declare.

Ethical Statement: The authors are accountable for all 
aspects of the work in ensuring that questions related to the accuracy or integrity of any part of the work are appropriately investigated and resolved. The study was conducted in accordance with the Declaration of Helsinki (as revised in 2013). The study was approved by Ethics Committee of Shandong Cancer Hospital (No. SDTHEC 20110324) and individual consent for this retrospective analysis was waived.

Open Access Statement: This is an Open Access article distributed in accordance with the Creative Commons Attribution-NonCommercial-NoDerivs 4.0 International License (CC BY-NC-ND 4.0), which permits the noncommercial replication and distribution of the article with the strict proviso that no changes or edits are made and the original work is properly cited (including links to both the formal publication through the relevant DOI and the license). See: https://creativecommons.org/licenses/by-nc-nd/4.0/.

\section{References}

1. Schrenk P, Shamiyeh A, Wayand W. Sentinel lymph-node biopsy compared to axillary lymph-node dissection for axillary staging in breast cancer patients. Eur J Surg Oncol 2001;27:378-82.

2. Wang Y, Yu J, Wang L. The study of sentinel node biopsy in clinical node negative breast cancer in China. San Antonio: 27th Annual Charles A Colman San Antonio Breast Cancer Symposium, 2004:88-9.

3. Morrow M, Dang CT. Sentinel node biopsy after neoadjuvant chemotherapy: a new standard for patients with axillary metastases? JAMA 2013;310:1449-50.

4. Kuehn T, Bauerfeind I, Fehm T, et al. Sentinel-lymphnode biopsy in patients with breast cancer before and after neoadjuvant chemotherapy (SENTINA): a prospective, multicentre cohort study. Lancet Oncol 2013;14:609-18.

5. Boughey JC, Suman VJ, Mittendorf EA, et al. Sentinel lymph node surgery after neoadjuvant chemotherapy in patients with node-positive breast cancer: the ACOSOG Z1071 (Alliance) clinical trial. JAMA 2013;310:1455-61.

6. Grasishar WJ, Andrson BO, Balassanian R, et al. Breast cancer, Version 22018 featured updates to the NCCN guidelines. National Comprehensive Cancer Network. Available online: http://www. NCCN.org

7. Curigliano G, Burstein HJ, Winer EP, et al. De-escalating and escalating treatments for early-stage breast cancer: the St. Gallen international expert consensus conference on the primary therapy of early breast cancer 2017. Ann
Oncol 2017;28:1700-12.

8. Kantor O, Sipsy LM, Yao K, et al. A predictive model for axillary node pathologic complete response after neoadjuvant chemotherapy for breast cancer. Ann Surg Oncol 2018;25:1304-11.

9. Eisenhauer EA, Therasse P, Bogaerts J, et al. New response evaluation criteria in solid tumours: revised RECIST guideline (version 1.1). Eur J Cancer 2009;45:228-47.

10. Ersoy YE, Kadioglu H. Review of novel sentinel lymph node biopsy techniques in breast cancer patients treated with neoadjuvant chemotherapy. Clin Breast Cancer 2018;18:e555-9.

11. Bonnefoi H, Litière S, Piccart $M$, et al. Pathological complete response after neoadjuvant chemotherapy is an independent predictive factor irrespective of simplified breast cancer intrinsic subtypes: a landmark and two-step approach analyses from the EORTC 10994/BIG 1-00 phase III trial. Ann Oncol 2014;25:1128-36.

12. Rescigno J, Zampell JC, Axelrod D. Patterns of axillary surgical care for breast cancer in the era of sentinel lymph node biopsy. Ann Surg Oncol 2009;16:687-96.

13. Buzdar AU, Ibrahim NK, Francis D, et al. Significantly higher pathologic complete remission rate after neoadjuvant therapy with trastuzumab, paclitaxel, and epirubicin chemotherapy: Results of a randomized trial in human epidermal growth factor receptor 2-positive operable breast cancer. J Clin Oncol 2005;23:3676-85.

14. Hennessy BT, Hortobagyi GN, Rouzier R, et al. Outcome after pathologic complete eradication of cytologically proven breast cancer axillary node metastases following primary chemotherapy. J Clin Oncol 2005;23:9304-11.

15. Weber JJ, Jochelson MS, Eaton A, et al. MRI and Prediction of Pathologic Complete Response in the Breast and Axilla after Neoadjuvant Chemotherapy for Breast Cancer. J Am Coll Surg 2017;225:740-6.

16. Kim WH, Kim HJ, Park HY, et al. Axillary Pathologic Complete Response to Neoadjuvant Chemotherapy in Clinically Node-Positive Breast Cancer Patients: A Predictive Model Integrating the Imaging Characteristics of Ultrasound Restaging with Known Clinicopathologic Characteristics. Ultrasound Med Biol 2019;45:702-9.

17. Vila J, Mittendorf EA, Farante G, et al. Nomograms for Predicting Axillary Response to Neoadjuvant Chemotherapy in Clinically Node-Positive Patients with Breast Cancer. Ann Surg Oncol 2016;23:3501-9.

18. Bilimoria KY, Stewart AK, Winchester DP, et al. The National Cancer Data Base: a powerful initiative to improve cancer care in the United States. Ann Surg Oncol 
2008,15:683-90.

19. Gentilini O, Veronesi U. Abandoning sentinel lymph node biopsy in early breast cancer? A new trial in progress at the European Institute of Oncology of Milan (SOUND: Sentinel node vs Observation after axillary UltraSouND). Breast 2012,21:678-81.

20. Flores-Funes D, Aguilar-Jiménez J, Martínez-Gálvez M, et al. Validation of the targeted axillary dissection technique in the axillary staging of breast cancer after neoadjuvant therapy: Preliminary results. Surg Oncol 2019,30:52-7.

Cite this article as: Zheng W, Zhou P, Liu Y, Liang Y, Wang Y. Prediction of axillary response after neoadjuvant chemotherapy in clinical node positive breast cancer. Transl Cancer Res 2021;10(6):2822-2830. doi: 10.21037/tcr-20-3454
21. Kanesalingam K, Sriram N, Heilat G, et al. Targeted axillary dissection after neoadjuvant systemic therapy in patients with node-positive breast cancer. ANZ J Surg 2020;90:332-8.

22. Simons JM, van Nijnatten TJA, van der Pol CC, et al. Diagnostic Accuracy of Different Surgical Procedures for Axillary Staging After Neoadjuvant Systemic Therapy in Node-positive Breast Cancer: A Systematic Review and Meta-analysis. Ann Surg 2019;269:432-42. 